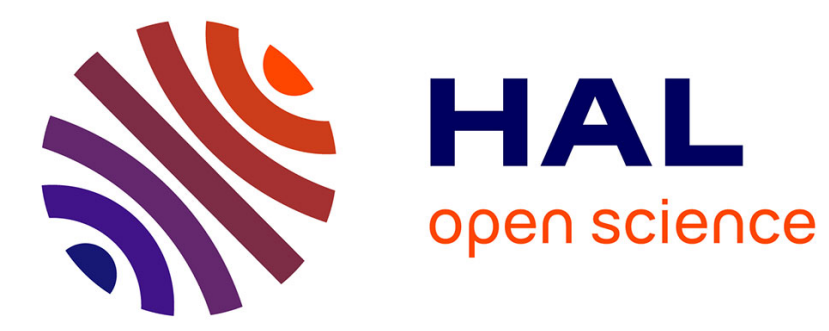

\title{
Approche anthropologique des interdits sexuels autour de la mort
}

\author{
Bernadette Tillard
}

\section{To cite this version:}

Bernadette Tillard. Approche anthropologique des interdits sexuels autour de la mort. Etudes sur la mort. Revue de la Société de thanatologie, 2015, 147 (1), 10.3917/eslm.147.0125 . halshs-01228867

\section{HAL Id: halshs-01228867 https://shs.hal.science/halshs-01228867}

Submitted on 24 Nov 2015

HAL is a multi-disciplinary open access archive for the deposit and dissemination of scientific research documents, whether they are published or not. The documents may come from teaching and research institutions in France or abroad, or from public or private research centers.
L'archive ouverte pluridisciplinaire HAL, est destinée au dépôt et à la diffusion de documents scientifiques de niveau recherche, publiés ou non, émanant des établissements d'enseignement et de recherche français ou étrangers, des laboratoires publics ou privés. 
Bernadette TILLARD,

Professeure

CLERSE - UMR 8019

Bâtiment SH2, bureau 201, cité scientifique,

59655 Villeneuve d'Ascq Cedex

bernadette.tillard@univ-lille1.fr 


\section{Résumé :}

Les interdits sexuels au moment de la mort s'inscrivent dans un ensemble de restrictions concernant les proches durant la phase intermédiaire (marge) du rite de passage. D'autre part, si peu d'anthropologues ont précisé ces interdits, les coutumes concernant le deuil des veuves et leur remariage nous donnent indirectement un aperçu des préconisations et de leurs fonctions, inscrivant l'interdit sexuel dans une problématique plus vaste et collective de cycle de vie.

\section{Mots-clés :}

Sexualité, mort, anthropologie, reproduction.

\section{Summary:}

Sexual prohibitions at the time of death are part of restrictions on relatives during the middle phase (margin) of the rite of passage. On the other hand, if few anthropologists wrote about these prohibitions, indirectly, customs regarding mourning and remarriage of widows give us a glimpse of the recommendations and their functions, embedding the sex bans during mourning in the broader issue of life cycle.

\section{Keywords:}

Sexuality, death, anthropology, reproduction. 


\section{Approche anthropologique des interdits sexuels autour de la mort}

\section{Introduction}

Dans le domaine de la mort, comme dans celui de la naissance, nos repères ont été bouleversés depuis le début du siècle dernier. C'est sous surveillance médicale que l'homme laisse échapper un dernier soupir et que le nouveau-né pousse son premier cri. Les lieux se sont diversifiés, les acteurs également. La maison, la famille élargie et le voisinage ne sont plus les seuls entourages mobilisés lors de ces événements. Actuellement, environ $58 \%$ des personnes décèdent en milieu hospitalier, tandis que $27 \%$ meurent à domicile (Lalande \& Veber, 2009). Par ailleurs, même si les morts prématurées n'ont pas disparues, nos contemporains ont une longévité plus grande. Le texte proposé ne repose pas sur une étude empirique, mais il s'inspire d'une présentation orale effectuée en 2001 (Tillard, 2001) sur la base de lectures dont certaines références issues des Human Relations Areas Files $(H R A F)$ consultées au Laboratoire d'Anthropologie Sociale et plus particulièrement celles intitulées General sex restrictions, Purification and expiation, secundary mariages, dependancy (care of widows).

Le contexte qui préside aux rites de deuil a donc beaucoup évolué. Les rites, ceux de deuil comme ceux de naissance, sont l'occasion de cérémonies et de rencontres qui permettent de marquer les étapes de la vie humaine. Face aux événements heureux ou malheureux, ils offrent un cadre aux personnes et aux groupes pour partager leur expérience et la jalonner de ces moments collectifs. Les séparations (séparation du défunt et de sa famille lors du décès/ séparation du fœetus et de la femme lors de la naissance) étaient suivies d'une période de mise à l'écart des proches d'une durée fixée par la tradition et variable selon la localité, puis d'un moment d'intégration (pour le défunt et pour le nouveau-né) et de réintégration (pour les endeuillés et la mère). Séparation, mise à l'écart et 
intégration constituent une séquence ordonnée et nommée «rites de passage » par Arnold Van Gennep au début du XX $X^{\mathrm{e}}$ siècle (Van Gennep, 1909).

Cependant dès cette étape de la réflexion, un fait majeur s'impose : autrefois, la séparation - dans un cas le décès, dans l'autre l'accouchement - marquait le début d'un long processus de prise en charge du défunt et de son entourage. Or, si nous considérons nos propres manières d'agir depuis la fin du $\mathrm{XX}^{\mathrm{e}}$ siècle, nous devons constater que l'investissement social s'est déplacé de l'aval vers l'amont, pour privilégier les moments qui précèdent la séparation au détriment de ceux qui lui succèdent. Les débats publics et parlementaires sur la prise en charge de la douleur, les soins palliatifs et la fin de vie, même s'ils donnent voix à ceux qui voudraient une réponse plus complète aux attentes sociales quant à la prise en charge des personnes mourantes, témoignent par leur présence, leur fréquence et par l'écho dans les médias, de l'importance de cette préoccupation sociale, tandis que le deuil ne fait guerre l'objet de discours à l'exception des décès médiatisés car survenant de manière violente ou touchant une célébrité. De même, le suivi minutieux des grossesses, les politiques publiques de prévention des accidents domestiques ou de travail, les discours sur l'hygiène alimentaire, les méfaits du tabac ou la modération de la consommation d'alcool, ces domaines tout comme la prise en charge médicale de la maladie sont aujourd'hui autant de dispositifs qui cherchent à intervenir avant la naissance et à éviter ou à différer la maladie et la mort.

Dans un cas comme dans l'autre, la polarité de l'investissement social s'est inversée : la période qui précède la séparation fait l'objet de toute l'attention de la médecine pour que le moment du décès survienne plus tardivement et dans des conditions si possible moins douloureuses. Le déroulement temporel est donc centré sur cet avant. Le départ rapide du corps du défunt, du service hospitalier vers le funérarium, confirme l'impression qu'une fois l'événement advenu, tout doit aller très vite : « la conservation du corps en chambre ne peut excéder dix heures » (Lalande \& Veber, 2009, p. 79). Les politiques de raccourcissement du séjour maternel en milieu hospitalier vont dans le même sens. Depuis la fin du $\mathrm{XX}^{\mathrm{e}}$ siècle, la mort comme la naissance font donc l'objet d'attention soutenue et fortement médicalisée et hospitalière, avec un intérêt prépondérant pour ce qui précède le moment de 
la séparation. L'évolution des pratiques sociales ne saurait être le fruit du hasard, mais est le reflet de modifications profondes de notre manière de penser les événements. Dans ce contexte, réfléchir sur les interdits sexuels autour de la mort peut sembler anachronique. Pourquoi ces pratiques existaientelles ? Quels sens et quels rôles pouvaient-elles avoir? Que nous disent-elles du sens attribué à la mort?

\section{Les interdits sexuels}

Sans doute est-il utile de tenter de situer les interdits sexuels parmi les autres formes d'interdits. Dans Les Formes élémentaires de la vie religieuse, Émile Durkheim présente les différents interdits comme constituant un culte négatif (Durkheim, 1912, p. 431). Il distingue l'interdit magique dont la transgression est sanctionnée uniquement par des conséquences physiques ou matérielles, de l'interdit religieux appelant non seulement une conséquence palpable mais également un blâme, une réprobation publique(Durkheim, 1912, p. 429). Les interdits réalisent la séparation des êtres sacrés et des êtres profanes. Durkheim précise : «Tout un ensemble de rites a pour objet de réaliser cet état de séparation qui est essentiel. Puisqu'ils ont pour fonction de prévenir les mélanges et les rapprochements indus, d'empêcher qu'un de ces deux domaines n'empiète sur l'autre, ils ne peuvent édicter que des abstentions, c'est-à-dire des actes négatifs. Pour cette raison, nous proposons d'appeler culte négatif le système formé par ces rites spéciaux. Ils ne prescrivent pas au fidèle d'accomplir des prestations effectives, mais se bornent à lui interdire certaines façons d'agir ; ils prennent donc tous la forme de l'interdit, ou, comme on dit couramment en ethnographie, du tabou » (Durkheim, 1912, p. 429). S'en suit l'énumération des formes multiples de ces interdits : interdit de contact, interdit alimentaire, interdit de toucher, interdit de parole, interdit d'écoute, interdit vestimentaire, interdit de certains actes ordinaires (manger), interdits temporels (découpage du temps en jours ordinaires et jours de fête). Certains de ces interdits sont retrouvés en association aux interdits sexuels dans les textes concernant la naissance et la mort.

Les interdits sexuels auxquels nous nous intéressons surviennent après le décès d'un proche. Durant 
cette période, le défunt et les endeuillés sont tenus momentanément à l'écart de la société. Cette mise à l'écart des activités ordinaires est un statut provisoire et partagé par toutes les personnes, à des moments différent de leur histoire, selon les événements auxquels elles sont confrontées.

\section{Eviter le contact avec des êtres en transition}

Durant cette période de marge qui suit le décès, l'interdit sexuel est d'abord à comprendre comme un interdit de contact parmi d'autres qui concerne les personnes faisant l'objet d'un rite de passage. C'est-à-dire celles dont le statut est temporairement indéfini. Le défunt et ses proches, tout comme le fœtus et celle qui le porte sont dans des positions intermédiaires, difficiles à cerner. Cette imprécision ne permet pas de leur assigner une place ordinaire dans la société. Ils sont considérés simultanément comme des êtres dangereux et vulnérables. Comme le souligne Mary Douglas, on leur associe fréquemment le caractère d'impureté que les seules considérations hygiénistes ne sauraient justifier (1) (Douglas, 1992).

La protection qui leur est accordée se traduit dans les précautions que l'on prend à leur égard, mais ces attentions doivent être vues à la fois comme une protection qu'on leur assure dans un moment de fragilité, mais également comme une protection que l'ensemble de la société assure à ses membres en codifiant leur relations avec ceux qui sont touchés très directement par le deuil.

\section{Se protéger du défunt et des proches}

Le défunt se situe à un moment de l'histoire qui lui donne un statut particulier : le corps repose inerte n'a pas encore totalement renoncé à son commerce avec les vivants. De même que le nouveau-né n'est pas encore vraiment ancré dans la vie terrestre, l'un et l'autre, défunt et nouveau-né sont considérés comme ayant des transactions tant avec les humains qu'avec l'au-delà. A ce titre, ils sont craints. Le défunt va-t-il accepter son départ et entretenir des relations paisibles avec les vivants ? Au contraire, sera-t-il à jamais jaloux de la longévité des survivants et de leurs richesses ? Le nouveau-né va-t-il s'accrocher suffisamment à la vie pour grandir et devenir adulte ? L'esprit qui l'habite va-t-il 
être satisfait de cette incarnation ? L'un et l'autre sont perçus comme ayant un long chemin à parcourir l'un pour quitter la vie et devenir ancêtre protecteur, l'autre pour renoncer à l'au-delà et habiter la terre.

Les interdits sexuels au moment d'une naissance et d'un décès sont à comprendre dans ce contexte où la vie de l'individu est perçue comme une entité négociée avec l'au-delà. La contagiosité (Durkheim) de l'état de transition dans lesquels sont le défunt et le nouveau-né sont également à la source d'une autre inquiétude : les esprits vont-ils profiter de ces êtres entre deux états pour s'emparer de quelques autres vivants ? Cette inquiétude concerne essentiellement ceux qui vivent sous le même toit que le défunt ou la mère du nouveau-né. Aussi, les sociétés tentent-elles de se protéger de ceux-là en les associant à la marginalité du défunt et du nouveau-né dans un objectif de double protection : protection des proches à l'égard de l'extérieur, protection de l'extérieur vis-à-vis des proches.

Cette protection par la mise à l'écart des activités ordinaires et la limitation des déplacements est rattachée au temps du biologique : dans le cas du défunt, il s'agit du devenir du corps (la mise en terre puis la décomposition ou le dessèchement du corps). Dans le cas du nouveau-né, il s'agit de l'étape terminale de l'accouchement (temps d'écoulement des lochies) et de maturation du corps de l'enfant (première dent, sevrage).

Nous pensons donc que les interdis qui frappent le défunt et les proches sont une modalité de mise à distance à vocation préventive à l'égard de ceux qui se trouve à l'extérieur de ce premier cercle. Cependant, les proches ne sont pas laissés seuls pour accomplir les tâches nécessaires au traitement du corps du défunt.

\section{Prendre soin du défunt}

En effet, selon les circonstances et les sociétés, le cadavre est tantôt traité avec bienveillance, attention et respect, tantôt l'objet de persécution, de manipulations violentes. La préparation du corps est un élément codifié qui, à l'intérieur d'une société donnée, dépend du statut hiérarchique de la personne, de son statut matrimonial, de son âge et des circonstances de son décès. Ainsi les décès 
prématurés de femmes en couche sont parfois associés à des traitements particuliers et violents du cadavre.

Souvenons-nous également dans notre propre civilisation de l'exposition du cadavre gravement mutilé des condamnés à mort. Cette exposition venait prolonger sa désapprobation par delà la mort. A l'opposé de la vision irrespectueuse d'un corps supplicié, Yvonne Verdier donne la parole à la « femme-qui-aide » qui exprime le souci d'apporter un soin particulier à la toilette et la présentation du défunt : "On m'appelle dès qu'ils sont morts. Il faut laver le mort, le raser si c'est un homme, le coiffer bien. Ensuite, je l'habille, en principe avec quelque chose de propre [Les gens âgés tiennent prêt dans l'armoire leur habit de cérémonie, le costume de mariage ou la robe de lendemain de noce]. Quand le mort est prêt, bien habillé, il faut un homme pour le soulever, le mettre au fauteuil pendant que je prépare le lit... On met un drap propre sur le lit d'où on a tout enlevé, sauf le sommier ; fallait prendre un drap, pas un drap neuf, non, un drap qui soit assez souple... Ensuite on installe le mort sur le lit, on lui ferme les yeux, on lui ferme la bouche. On lui cache le visage avec une serviette ou un mouchoir blanc; les mains, on lui croise sur le ventre, et on lui met un chapelet dessus avec un brin de buis. On le couvre ensuite avec un autre drap blanc, un joli drap, bien fin, on le plisse bien. Pour le mettre au cercueil, on ôte le drap de dessus et on rabat celui du dessous par-dessus » (Verdier, 1979, p. 101). La délicatesse de ces soins transparaît dans la minutie des gestes décrits et dans certains détails comme la nature du drap de dessous, celui choisit pour couvrir le mort jusque dans le cercueil. Ce drap doit être souple. Il faut entendre par là que ce n'est pas un drap neuf de toile épaisse, mais un drap qui doit avoir été assoupli, usé par de multiples usages. C'est dans cette matière douce au toucher que l'on fait tant le drap du mort que le linge de corps du nouveau-né.

Dans la présentation du cadavre, la question des objets qui l'accompagnent est souvent posée. Ici, le chapelet, l'eau bénite et le brin de buis pour les hommes comme pour les femmes. Là-bas, les objets utilisés ou leur représentation en miniature, objets propres à chaque sexe accompagnent le défunt dans sa tombe ou sur le bûcher, soulignant ses attributs identitaires. Rasmussen à propos des Inuits précise que l'homme décédé doit toujours être enseveli avec les objets au moyen desquels il fait son voyage sur terre : son harpon, ses couteaux, ses flèches etc. Si ces objets sont de grande valeur, ils 
seront remplacés par des objets plus communs ou des objets miniatures. Teit chez un peuple indien d'Amérique du Nord (les Nahanes) fait la même observation : «certains des objets appartenant au défunt qui était brûlé avec lui étaient placés près du corps ».

Cette pratique n'est pas rare, qui attribue au défunt certains objets qui accompagnent le corps dans ce qu'il advient de lui au-delà des cérémonies funéraires. Dans ces précisions, il est perceptible que le corps du défunt continue de porter son identité sexuée. Par «Le costume de mariage ou la robe de lendemain de noce », le défunt est particulièrement honoré comme homme ou femme, rappelant le moment de l'entrée dans une sexualité officielle et légitime à ceux qui viendront présenter leurs condoléances aux proches. Parmi les biens du défunt, certains le suivront donc par delà la mort, d'autres seront distribués aux héritiers, d'autres seront consommés lors des cérémonies de funérailles. Le sort réservé à son nom sera lui-aussi objet d'attention : convient-il de donner son nom à un prochain nouveau-né ou son nom sera-t-il éradiqué, interdit de prononciation ? Ce qui constituait la personne du défunt et lui était attribué personnellement va tantôt être caché, tantôt rester présent et connaître d'autres usages.

\section{Prendre soin des proches}

Chez Yvonne Verdier, l'attention au défunt s'accompagne de celle accordée aux proches. «Le mort bien arrangé, la femme-qui-aide se livre à tout un ménage mortuaire : fermer les fenêtres, les volets ...». L'âme du défunt doit être évacuée avec l'eau de la toilette mortuaire et ne pas être piégée par les reflets qui pourraient l'induire en erreur durant son cheminement. "La femme-qui-aide calfeutre tout, interdit toute activité domestique dans la maison, tout remue-ménage pouvant déranger dans son parcours ; enfin elle couvre le miroir et la télévision » (Verdier, 1979, p. 139).

Plus généralement, dans les descriptions ethnographiques, les endeuillés sont décrits comme brutalement interrompus dans leurs activités par la mort d'un proche, revêtant des vêtements ou des maquillages particuliers, exprimant la tristesse. Ne bougeant pas de la maison, ils sont relayés par des voisins ou des parents dans leurs tâches ordinaires. Les personnes touchées de près par le deuil sont 
distinguées du reste du groupe et entravées dans leurs mouvements. Ainsi, Chabas chez les Wolofs du Sénégal, présente les veuves «obligées de rester accroupies et sans mouvement dans la case où était le défunt jusqu'à ce que la sœur du mari vienne leur dénouer les cheveux et faire leur toilette de deuil »(HRAF, Chabas, MS30, Wolof, p. 42-43). L'intensité du deuil est proportionnelle à la proximité de parenté entre le sujet et le défunt.

L'explicitation, relativement rare, de l'interdit sexuel tient probablement au fait que les auteurs considèrent que cela va de soi. Le comportement attendu étant l'expression du chagrin, il n'est pas question de se distraire de cette injonction par un plaisir charnel. De plus, la restriction dans les déplacements, voire l'immobilité, vécue durant cette phase liminaire, vient construire un contexte favorable au respect de l'interdit.

\section{Expression codifiée des émotions de la veuve}

Parmi les personnes dont le statut est souvent abondamment décrit, il y a la veuve. Dans Mours et coutumes des Maoris, écrit en 1938, Thomas Edward Donne décrit ainsi des pratiques de deuil particulièrement démonstratives : "La veuve du chef affichait un chagrin violent avec des pleurs et des lamentations excessives, se coupant les joues la poitrine et les bras, jusqu'à faire couler le sang abondamment, chaque lacération sérieuse s'accompagnant d'un hurlement à faire figer le sang dans les veines. Ces entailles se faisaient avec des éclats d'obsidienne aussi coupants que des fragments de verre ou avec des morceaux de jade aiguisés jusqu'à avoir un bord tranchant comme un rasoir.

J'ai vu des veuves se livrer à la cérémonie des lacérations, et leur sang couler à flot des entailles longues et relativement profondes qu'elles se faisaient. Lorsque la première explosion de douleur était calmée et que la veuve avait le sentiment d'avoir été à la hauteur des circonstances, en sauvegardant la dignité de son mari défunt, elle cessait de se blesser, le sang coagulait et séchait sur les cicatrices en voie de guérison....

Parfois les cicatrices de deuil étaient colorées à dessein avec une teinture utilisée pour le tatouage, [et appelé ngaragu] ; elles devenaient ainsi le témoignage permanent de fidélité conjugale. Si 
l'exaltation de la veuve du chef était extrême, elle décidait parfois d'accompagner son mari dans son voyage suprême vers l'au-delà et elle attentait à sa propre vie en s'étranglant, ce qui était considéré comme le procédé le plus tika (correct) pour les dames de haut rang. »(Donne, 1938, p. 53)

La mort d'un chef donne souvent lieu à des cérémonies particulières mais le statut des femmes ayant perdu leur mari connaît rarement de telles exigences sociales. Plus souvent les veuves passent par une période de réclusion dans leur belle-famille.

\section{Explicitation d'un interdit sexuel touchant la veuve}

Pöli, femme pygmée, précise que durant le deuil, « une femme ne peut pas coucher avec un homme en plein jour et l'homme ne peut pas la toucher» (Donne, 1938, p. 53). C'est le rite de la coupe des cheveux qui met fin à la période de deuil dans un délai imprécis qui varie selon la disponibilité des ressources matérielles nécessaires à la fête qui s'en suit. Cette coupure des cheveux, à l'image de ce qui est pratiqué lors de certains baptêmes, renvoie à la symbolique d'une césure du temps, d'une rupture avec l'univers antérieur.

Certains ethnologues mentionnent brièvement cet interdit. Ainsi Barbara Glowczewski signale que, chez les Warlpiri, population d'aborigènes australiens, en ce qui concerne la sexualité, elle est interdite aux veuves avant la fin du deuil. Mais cet interdit n'est pas isolé. C'est tout un faisceau de restrictions qui frappent la veuve et transforme sa vie quotidienne: «Par ailleurs, les veuves ne peuvent ni manger de viande ni cuisiner, étant nourries par d'autres femmes »(Glowczewski, 1991, p. 197). Elles pratiquent également pendant le deuil le langage gestuel, appelé langage "main-main". L'interdit sexuel s'accompagne donc d'interdits alimentaires et d'interdit de langage. Elle souligne que les deuils successifs conduisent certaines femmes à passer une part non négligeable de leur existence sans prononcer un mot. Cette privation de paroles produit une forme de similitude avec le statut du défunt qui se trouve, lui aussi, empêché de s'exprimer.

Pour Louis-Vincent Thomas, l'interdiction aux endeuillés les relations sexuelles est d'une certaine manière leur faire partager un peu de la mort qui habite leur maison. Les endeuillés partageraient 
ainsi l'impossibilité de jouissance du défunt. Il voit également dans cet ensemble d'embarras créés par la mort, une manière de libérer les proches de la culpabilité de survivre, de la culpabilité de n'avoir pu éviter la souffrance et la mort de celui qu'ils pleurent (Thomas, 1982, p. 40-44).

\section{Asymétrie veuve-veuf}

A la lecture de nombreux textes au sujet de la mort et du deuil, une remarque vient à l'esprit. Les textes évoquent rarement le veuf tandis qu'ils s'attardent sur la veuve. Bien entendu, la description spectaculaire du deuil des veuves de chefs maoris ne pouvait avoir son corollaire puisque la chefferie

revenait à un homme. Mais cette asymétrie est présente dans d'autres circonstances. Quand l'auteur prend le temps de nous préciser la durée du deuil, la durée est plus brève pour l'homme que pour la femme. De plus, il est rarement mentionné les prescriptions qui touchent un mari ayant perdu sa femme alors que dans le cas inverse nous avons force détails.

Il est donc perceptible que les restrictions pour la veuve sont plus sévères que pour le veuf. Teit à propos des Nahanes, tribu indienne d'Amérique du Nord explique que «très peu de restrictions concernent les veufs. Certains jeûnent et vivent à l'écart pendant une courte période, jamais plus de six mois. Ces observances sont toutes optionnelles et la plupart ne les observent pas. Tous les veufs sont (cependant) interdits de chasse pendant un an. Cette restriction est généralement observée » (HRAF, Teit, Nahanes, ND12, p. 158-162). En revanche, toutes les veuves sont recluses, interdites de cueillette mais elles sont également privées de toilette, de coiffure, de chant et de danse. L'ensemble des viandes et poissons frais leur est interdit pendant un an tandis qu'elles devront se passer de certaines viandes pendant quatre ans. De même, Béranger-Ferand effectuant ses recherches au Sénégal précise que les veuves "gardent en guise de deuil un pagne roulé en turban autour de la tête pendant 4 mois... Le mari, de son côté, porte le deuil de sa femme pendant 8 jours seulement » (HRAF, Béranger-Ferand, Wolof, p. 42-43). 


\section{Délai de viduité}

Nous pouvons avoir une idée de l'intensité de ces interdits en prenant en considération le lévirat et le sororat et le remariage des veufs et des veuves. Cependant, il nous faudra garder à l'esprit que dans ce cas on parlera d'une période d'interdit matrimonial et non d'interdit sexuel et que nous ne serons renseignés uniquement pour les personnes les plus proches du défunt.

Fayet, chez les Wolofs, confirme qu'en cas de décès du mari, la veuve ne peut pas se remarier avant la fin de la période de réclusion de quatre mois et dix jours tandis qu'en cas de décès de sa femme, le mari qui n'est d'ailleurs pas appelé "veuf" par l'auteur mais bien "mari" peut contracter de nouveau un mariage immédiatement après le décès de sa femme. Un autre auteur encore, Chabas, toujours dans le même peuple nous fait remarquer que pour la femme, «Le délai de viduité est en principe de trois mois mais il est compté par périodes menstruelles. On compte un mois avec l'apparition des premières règles et les trois mois sont écoulés à la fin des troisièmes » (HRAF, Chabas, Wolof, MS30). Il remarque que ce délai est identique à celui prescrit en cas de divorce et que au-delà de la décence et de la convenance à ne pas se remarier trop tôt après la mort du conjoint, les raisons évoquées pour différer le remariage au-delà de trois mois sont les mêmes : il s'agit d'éviter le mariage de femmes enceintes.

Le contrôle social exercé sur la sexualité dans le cadre du deuil est donc à comprendre non seulement ainsi que nous l'avons évoqué précédemment, comme au regard d'un interdit de contact remplissant une fonction préventive de séparation, mais dans le cadre plus général de la fertilité féminine. La plupart des sociétés décrites par l'anthropologie, associant au moins partielle sexualité et procréation, l'asymétrie des interdits qui pèsent sur le veuf et la veuve évoque la question du statut de la veuve, et plus particulièrement de l'enfant dont elle serait enceinte. Les relations sexuelles durant la période de deuil et le mariage immédiatement après la mort du mari poseraient la question de l'appartenance de l'enfant à naître. A quelle famille serait affilié l'enfant qui serait issu d'une relation sexuelle estimée proche du décès ? A l'une ou l'autre de ses belles-familles, celle de son époux défunt ou celle de son nouveau-mari ? L’interdit sexuel après le décès du mari durant une période d'environ quatre mois 
vient apporter la possibilité d'observer une éventuelle grossesse contractée avant la mort du mari et dont le fruit reviendrait sa famille. En cas d'absence de grossesse perceptible durant ce délai, un enfantement qui surviendrait par la suite, serait attribué à une relation ultérieure.

\section{Interdit sexuel et parenté}

La question de l'affiliation d'un enfant issu d'une grossesse survenant chez la veuve, à proximité d'un deuil nous ramène à Durkheim. En effet, si celui-ci citaient les interdits de contact parmi les « rites ascétiques », ils écartait ceux qui nous intéressent plus particulièrement, les interdits sexuels, par une simple note de bas de page : Il y a une interdiction dont nous ne disons rien parce que la nature exacte n'en est pas très facilement déterminable : c'est le contact sexuel. Il y a des périodes religieuses où l'homme ne doit pas avoir commerce avec la femme. Est-ce parce que la femme est profane ou parce que l'acte sexuel est un acte redouté ? La question ne peut être tranchée en passant. Nous l'ajournerons comme tout ce qui concerne les rites conjugaux et sexuels. Ils tiennent trop étroitement au problème du mariage et de la famille pour en pouvoir être séparés (Durkheim, 1992, p.434).

Cette remarque corrobore les observations ultérieures en situant l'interdit sexuel lors d'un deuil dans le domaine de la parenté. Il souligne aussi combien l'objet de notre propos -les interdits sexuels autour de mort - procède d'un découpage arbitraire qui pourrait être envisagé autrement. Ainsi, nous pourrions envisager une présentation sur les interdits sexuels aux différents moments de la vie comme une forme sociale de maîtrise de la fertilité. Les interdits sexuels au moment du décès seraient alors été traités plutôt comme une forme particulière de non-accès à la procréation. La comparaison entre rite de naissance et rite de décès reprend ici toute sa pertinence.

\section{La femme-qui-aide : la question de son âge}

Des personnages décrits par l'ethnologie rendent possibles un rapprochement d'ordre symbolique entre mort et naissance, entre mort et fertilité. Ainsi, celle que l'on nommait "la femme-qui-aide", décrite par Yvonne Verdier, lave le mort, le prépare, organise la vie de la maisonnée durant les jours 
qui précèdent l'enterrement, etc. Son rôle n'est pas moins important lors de la naissance. Elle y assiste, lave le nouveau-né, le porte sur les fonds baptismaux, est présente lors du sevrage de l'enfant. Cette figure a perduré jusqu'à la fin de la seconde guerre mondiale.

Or, la femme-qui-aide à la toilette des nouveau-nés et des défunts n'est pas choisie au hasard. Alors que le bébé comme le mort sont l'objet de craintes, cette femme semble ne pas être en danger face à ces événements troublants. La question de son âge est peut-être au centre de son aptitude à faire les bébés autant que les morts : elle n'est plus d'un âge à faire jaser. Sans être explicitement soumise à des interdits sexuels, une partie de la compétence qu'on lui accorde est probablement liée à cette position un peu à l'écart des années traditionnellement reconnues comme sexuellement les plus actives. Elle est ici dans un rôle de passeur entre les êtres en position indécise et les autres personnes de la communauté. Elle agit avec assurance, elle orchestre les petites comme les grandes choses qui socialisent les deux bornes de l'existence humaine.

\section{Mort et fertilité}

Bien des sociétés en effet, mêlent de multiples façons la question de la mort et celle de la fertilité. Ce n'est pas seulement au travers de la question des interdits sexuels que cette question est abordée. Ainsi, Carmen Bernand à l'article mort du dictionnaire de l'ethnologie et de l'anthropologie souligne qu'en bien des cas les récoltes agricoles sont liées au statut et au devenir du corps des défunts. «Dans la mesure où la plupart des sociétés traditionnelles ont une conception cyclique du temps, la mort est associée à la fertilité. Parfois, le lien entre la dépouille humaine et la fécondité agraire est négatif. Les Moundang du Tchad croient que le cadavre du roi stérilise la terre (Bernand, 1991, p. 489) ». Dans ce cas, comme chez les Maoris (Donne, 1938), la pratique d'ensevelissement n'est pas de mise contrairement à ce qui est envisageable lorsque comme corps du défunt et fertilité sont associés «Dans le sud du Pacifique, l'apparition des plantes alimentaires à partir du corps de l'ancêtre est un thème fréquent. Au Pérou également, un même terme désignait l'ancêtre momifié et la

graine ». Ces citations de Carmen Bernand élargissent cette question des liens entre deuil et interdits 
sexuels à celle du cycle de la vie, à la succession des générations et à la préservation de la nourriture nécessaire à l'alimentation des hommes et des femmes.

Les coutumes interdisant momentanément les rapports sexuels à l'occasion de la naissance et de la mort, loin d'être des observances, fruits du hasard et de représentations isolées s'inscrivent dans un réseau signifiant qui dit combien chaque société se soucie de la transmission, de la reproduction et de la préservation de la vie humaine. En Occident, la mortalité maternelle liée à la naissance a beaucoup baissée et la plupart des adultes meurent tardivement à un âge où la question de la reproduction n'est plus à l'ordre du jour. Cependant, les morts violentes persistent (suicide) et certaines causes se sont développées au cours du $\mathrm{XX}^{\mathrm{e}}$ siècle (accidents de la route) emportant des personnes jeunes, en pleine possession de leurs capacités de transmission de la vie. Mais, pendant le $\mathrm{XX}^{\mathrm{e}}$ siècle, nous avons développé les moyens de maîtriser les connaissances et les techniques d'intervention sur la reproduction (échographie, test ADN de paternité, contraception, AMP, etc.). Aussi, les motifs qui conduisaient à tenir à l'écart ou à interdire le contact sexuel ont perdu une partie de leurs raisons d'être. Nous voici donc maintenant renvoyés à des normes plus implicites de bienséance et de vécu émotionnel. Si elles ne sont pas codifiées, elles sont néanmoins présentes. Dans cette transformation, les fonctions socio-anthropologiques perdent de leur importance pour laisser place à une appréciation plus subjective et individuelle.

\section{Bibliographie}

Bernand Carmen (1991), article « Mort». In Bonte P., Izard M. Dictionnaire de l'ethnologie et de l'anthropologie. Paris, PUF, p. 489.

Boursier D., Pöli, mémoires d'une femme pygmée, Paris, L'Harmattan, 1996.

Donne T. E. Mours et coutumes des Maoris, Paris, Payot, 1938.

Douglas Mary, De la souillure, études sur la notion de pollution et du tabou, Paris, Edition la découverte, 1992, (original 1967).

Durkheim E. Les formes élémentaires de la vie religieuse, Paris, PUF, 1912, réédition de 1960.

Glowczewski B., Du rêve à la loi chez les Aborigènes, Mythes, rites et organisation sociale en Australie, Paris, PUF, 1991, p197.

Human Relations Area Files (HRAF). Paris, Laboratoire d'Anthropologie Sociale.

Izard M., Smith P. La fonction symbolique, essais d'anthropologie, Paris, Gallimard, 1979.

Jaulin R. La mort Sara : l'ordre de la vie et la pensée de la mort au Tchad, Paris, Plon, 1967. 
Lalande F, Veber O, La mort à l'hôpital, Paris, Inspection Générale des Affaires Sociales, 2009.

Thomas L. V. La mort africaine : idéologie funéraire en Afrique noire, Paris, Plon, 1982.

Tillard B. Approche anthropologique des interdits sexuels autour de la naissance et de la mort, Lille 2, septembre 2001, Colloque « Deuil et sexualité ».

Van Gennep A., Les rites de passage. Paris, Picard, 1909, (réédition 1981).

Verdier Y. Façons de dire, façons de faire, La laveuse, la couturière, la cuisinière, Paris, Gallimard, 1979.

Notes :

(1) Douglas, 1992, p. 55 «Quand nous aurons détaché la pathogénie et l'hygiène de nos idées de la saleté, il ne nous restera de celle-ci que notre vieille définition : c'est quelque chose qui n'est pas à sa place. Ce point de vue est très fécond. Il suppose, d'une part, l'existence d'un ensemble de relations ordonnées et, d'autre part, le bouleversement de cet ordre. La saleté n'est donc jamais un phénomène unique, isolé. Là où il y a saleté, il y a système. La saleté est le sous-produit d'une organisation et d'une classification de la matière, dans la mesure où toute mise en ordre entraîne le rejet d'éléments non appropriés. " 